\title{
Bias in FGFR1 signaling in response to FGF4, FGF8, and FGF9
}

Kelly Karl ${ }^{1}$, Nuala Del Piccolo ${ }^{1}$, Pavel Krejci ${ }^{2,3}$, and Kalina Hristova ${ }^{1^{*}}$

${ }^{1}$ Department of Materials Science and Engineering, Institute for NanoBioTechnology, and Program in Molecular Biophysics, Johns Hopkins University, Baltimore MD 21218

${ }^{2}$ Department of Biology, Faculty of Medicine, Masaryk University, 62500 Brno, Czech Republic

${ }^{3}$ Institute of Animal Physiology and Genetics of the CAS, 60200 Brno, Czech Republic

\begin{abstract}
FGFR1 signals differently in response to the FGF ligands FGF4, FGF8 and FGF9, but the mechanism behind the differential ligand recognition is poorly understood. Here, we use biophysical tools to quantify multiple aspects of FGFR1 signaling in response to the three FGFs: potency, efficacy, ligand-induced oligomerization and downregulation, and conformation of the active FGFR1 dimers. We show that FGF4, FGF8, and FGF9 are biased ligands, and that bias can explain differences in FGF8 and FGF9-mediated cellular responses. Our data suggest that ligand bias arises due to structural differences in the ligand-bound FGFR1 dimers, which impact the interactions of the FGFR1 transmembrane helices, leading to differential recruitment and activation of the downstream signaling adaptor FRS2. This study expands the mechanistic understanding of FGF signaling during development and brings the poorly understood concept of receptor tyrosine kinase ligand bias into the spotlight.
\end{abstract}




\section{Introduction}

Fibroblast growth factor receptor (FGFR) belongs to the family of receptor tyrosine kinases (RTKs), which signal via lateral dimerization in the plasma membrane to control cell growth, differentiation, motility, and metabolism (1-3). The four known FGFRs (FGFR1-4) are singlepass membrane receptors, with an N-terminal ligand-binding extracellular (EC) region composed of three Ig-like domains, a transmembrane (TM) domain, and an intracellular (IC) region that contains a juxtamembrane (JM) domain, a kinase domain, and a cytoplasmic tail (4). The crossphosphorylation of tyrosines in the activation loop of the FGFRs in the ligand-bound dimers is known to stimulate catalytic activity, resulting in the phosphorylation of secondary intracellular tyrosines, which recruit cytoplasmic effector proteins (5). These effector proteins, once phosphorylated by FGFRs, trigger downstream signaling cascades that control cell behavior (6, 7).

The four FGFRs signal in response to extracellular ligands with a beta trefoil fold, called fibroblast growth factors (FGFs) (8). As many as 18 different FGF ligands are known to bind to and trigger distinct cellular responses through FGFR1-4 (9-11). The diversity in FGF-FGFR interactions far exceeds that of other RTK systems, reflecting the morphogen role that FGF signaling plays in the development of many tissues and organs. FGFR1 is well known for its critically important role in the development of the skeletal system, and has been implicated in many cancers (12-16). One specific example of a developmental process controlled by FGFR1 is limb bud outgrowth $(17,18)$. The limb bud forms early in the developing embryo and consists of mesenchymal cells sheathed in ectoderm. The process starts as the mesenchymal cells begin to proliferate until they create a bulge under the ectodermal cells above. This is followed by the formation of the apical ectodermal ridge (AER) by the cells from the ectoderm, which specifies the proximal-distal axis of the limb, ensuring limb outgrowth $(17,18)$. These roles are carried out by several FGF ligands, including FGF4, FGF8, and FGF9, which are produced and secreted by the ectodermal cells in the AER and have similar spatio-temporal expressions. The ligands diffuse into the mesenchymal region adjacent to the AER, and act upon the mesenchymal cells which express FGFR1. This leads to the activation of signaling pathways downstream of FGFR1, promoting survival and proliferation of the mesenchymal cells.

Experiments involving genetic ablation of FGF4, FGF8 and FGF9 have led to distinct limb malformations (19). Thus, the actions of these ligands through FGFR1 are different, but the 
mechanism behind the differential response of FGFR1 to multiple ligands has not been investigated. More broadly, it is not known how a cell recognizes the identities of different FGF ligands, when bound to and signaling through the same FGFR. Here we sought to investigate the molecular mechanism behind differences in FGFR1 signaling in response to FGF4, FGF8, and FGF9.

\section{Results}

\section{Differences in FGF-induced FGFR1 oligomerization}

While most RTKs signal as dimers, it has been reported that under some conditions RTKs can form oligomers with different signaling capabilities $(20,21)$. Therefore, we considered the possibility that FGF4, FGF8, and FGF9 promote the formation of different types of FGFR1 oligomers in the plasma membrane. We thus assessed the association state of FGFR1, labeled with YFP, using fluorescence intensity fluctuations (FIF) spectrometry. The fluorophores were attached to the C-terminus of FGFR1 via a GGS flexible linker; this attachment has been used before and has been shown to not affect function (22). The FIF experiments utilized 293T cells stably transfected with FGFR1-YFP.

FIF calculates molecular brightness of the YFP-tagged receptors in small regions of the cell membrane (23). The molecular brightness, defined as the ratio of the variance of the fluorescence intensity within a membrane region to the mean fluorescence intensity in this region, is known to scale with the oligomer size (24). The molecular brightness distributions of monomeric (Linker for Activation of T-cells, LAT; gray) $(25,26)$ and dimeric controls (TrkA in the presence of 130 nM nerve growth factor, NGF; black) (27) in 293T cells are shown in Figure 1A, along with the brightness distribution for FGFR1 in 293T cells stably expressing FGFR1-YFP (red). We found that FGFR1, in the absence of ligand (red line), exists in a monomer/dimer equilibrium, as its brightness distribution is between the distributions of LAT (monomer control) and TrkA in the presence of saturating concentration of NGF (dimer control). The FIF experiments also report on the concentration of the receptors at the cell surface, which we find to be in the range 100-200 FGFR $1 / \mu \mathrm{m}^{2}$ in the stable cell line. This concentration is similar to previously reported FGFR expression levels of the order of $\sim 80,000$ receptors per cell, corresponding to $\sim 80-100$ receptors $/ \mu \mathrm{m}^{2}(28)$. 
Next, we performed FIF experiments in the presence of FGF4, FGF8, and FGF9. The three ligands were added at high concentrations (130 nM), which exceed the reported FGF binding constants (in the $\sim \mathrm{nM}$ range)(29, 30) such that all FGFR1 molecules are ligand-bound. The brightness distributions in the presence of the ligands are shown in Figure 1A. We see that the brightness distributions recorded in the presence of FGF8 (green) and FGF9 (blue) overlap with the distribution for the dimer control, while the distribution for FGF4 (orange) appears shifted to higher brightness. This suggests that the average oligomer size may be increased beyond a dimer in the presence of FGF4. We analyzed the likelihood of this possibility using a statistical test. Since the distributions of molecular brightness are log-normal, we analyzed the corresponding $\log$ (brightness) distributions which are Gaussian (Figure 1B). The parameters of the Gaussian distributions and the standard errors were calculated and used in a Z-test. Results, shown in Figure 1C, show that there are statistically significant differences $(Z>2,(31))$ between the FGFR1 brightness distribution in the FGF4 case and the distribution for the dimer control (TrkA+NGF). Likewise, there are statistically significant differences between the FGFR1+FGF4 brightness distribution, on one hand, and the FGFR1+FGF8 and FGFR1+FGF9 brightness distributions, on the other $(\mathrm{Z}>2)$. Further, the distributions measured for FGFR1+FGF8 and FGFR1+FGF9 are the same as the dimer control distribution. This analysis demonstrates that while FGF8-bound and FGF9-bound FGFR1 forms dimers, FGF4 binding also promotes the formation of higher order FGFR1 oligomers, in addition to FGFR1 dimers.

\section{Differences in FGFR1 Dose Response curves.}

Next, we studied FGFR1 signaling in response to FGF4, FGF8, and FGF9 using quantitative western blotting. In particular, we acquired FGFR1 dose response curves while varying the concentrations of FGF4, FGF8, or FGF9 over a broad range. As we sought mechanistic interpretation of the results, we used the same 293T cell line used in the FIF experiments, since the FGFR1 expression and the FGFR1 oligomer size in the cell line is known. In the experimental design, we also took into account the widely accepted view in pharmacology that biased signaling, or a ligand's ability to preferentially activate one pathway over another, will manifest itself in every cell line, as it is a property of the ligand-receptor pair (32-35). 
The cells were incubated with FGFs for 20 minutes at $37^{\circ} \mathrm{C}$, after which the cells were lysed, and the lysates were subjected to SDS-PAGE while probing with antibodies recognizing specific phospho-tyrosine motifs. We assessed several "responses": (i) phosphorylation of Y653/654, the two tyrosines in the activation loop of the FGFR1 kinase that are required for kinase activity (4, $6,36,37$ ); (ii) phosphorylation of Y766 in the kinase tail of FGFR1, which serves as a binding site for PLC $\gamma$ (38); (iii) phosphorylation of FRS2, an adaptor protein that is constitutively associated with FGFR1 through interactions that do not involve phosphorylated tyrosines (39, 40); and (iv) phosphorylation of PLC $\gamma$ which binds to Y766 (41). In addition, we also blotted for the total expression of FGFR1, thus assaying for ligand-induced FGFR1 downregulation. Typical western blots are shown in Figure 2A. The band intensities from at least three independent experiments were quantified and plotted for each response and each ligand concentration in Figures 2C. The dose response curves in each panel were placed on a common scale by rerunning some of the samples on a common gel, as shown in Figure 2B.

The quantified results are shown in Figure 2C, which reveals unexpected differences in the shape of the dose response curves. While FGF8 and FGF9 dose response curves appear sigmoidal when plotted on a semi-log scale, as expected for a rectangular hyperbolic curve (eqn 1), FGF4 exhibits an increase in phosphorylation up to $2.6 \mathrm{nM}$ ligand, followed by a marked decrease in phosphorylation as the FGF4 concentration is further increased, for all studied phosphorylation responses.

What could explain the very unusual FGF4 dose response curves in Figure $2 \mathrm{C}$ ? We first considered the possibility that the shape of the FGF4 dose response is due to strong FGF4induced FGFR1 degradation at high FGF4 concentrations. Data in Figure 2C, however, do not support this view as the effect of FGF4 on FGFR1 downregulation is smaller when compared to the effects of FGF8 and FGF9. Thus, differential ligand-induced FGFR1 degradation cannot explain the shape of the dose response curves.

We next considered the possibility that the shape of the FGF4 dose response curve is due to differential FGFR1 de-phosphorylation kinetics. In particular, we asked whether at high FGF concentration, a fast de-phosphorylation occurs for FGF4-bound FGFR1, while the dephosphorylation of FGF8-bound and FGF9-bound FGFR1 is slower. As the western blot data were acquired after 20 minutes of stimulation, such differences in kinetics could explain the 
shape of the dose response. We thus measured Y653/654 phosphorylation via western blotting as a function of time, when $130 \mathrm{nM}$ ligand was added to the cells for a duration of $0,1,5,10,20$, and 60 minutes. The averages of 3 independent experiments are shown in Figure 3A, along with the standard errors. We see a quick increase in phosphorylation over time, followed by a decrease which may be due to FGFR1 de-phosphorylation and/or FGFR1 downregulation. Notably, the phosphorylation decrease in response to FGF4 is very similar when compared to the decrease in the presence of FGF9, while the FGF8-induced phosphorylation decrease is smaller. Thus, the FGF4-induced de-phosphorylation kinetics are not fundamentally different when compared to the FGF9 results and cannot provide an explanation for the observed decrease in FGFR1 phosphorylation at high FGF4 concentration.

Another possible explanation for the shape difference in the dose response curves could be that the FGF4-stabilized FGFR1 oligomers, observed in the FIF experiments at high FGF4 concentration (Figure 1), are less active than the FGFR1 dimers. To gain further insights into FGF4-induced FGFR1 oligomerization, we performed FIF experiments in the presence of 2.6 nM FGF4, which corresponds to the peak of Y653/654 phosphorylation in the FGF4 dose response curve. The brightness distribution at $2.6 \mathrm{nM}$ FGF4, shown in Figure 1, lies between the monomer control and the dimer control. The Z-test analysis shows that the 2.6nM FGF4 brightness distribution is significantly different from both the monomer and dimer control distributions, as well as from the distribution observed in the presence of high FGF4 concentration. Thus, at low FGF4 concentration, FGFR1 exists primarily in monomeric and dimeric states, while higher FGF4 concentrations (>2.6 nM FGF4) induce the formation of FGFR1 oligomers. The increase in phosphorylation at low FGF4 concentration can therefore be associated with FGFR1 dimers, while the subsequent phosphorylation decrease can be correlated with the appearance of oligomers in addition to dimers. Thus, the assumption that FGFR1 oligomers are less active than FGFR1 dimers can indeed provide an explanation for the observed shape of the FGF4 dose response curves.

\section{Differences in Potencies, Efficacies, and Bias.}

We next analyzed the dose response curves in Figure $2 \mathrm{C}$ to determine the potencies and the efficacies of FGF4, FGF8 and FGF9. These two parameters were determined as optimal fit 
parameters in the context of rectangular hyperbolic dose response curves (see Eq. 1), as done in the GPCR literature (42-44). The efficacy (Etop in Eq.1) is the highest possible response that can be achieved for a ligand, typically at high ligand concentration. The potency (EC50 in Eq.1), on the other hand, is the ligand concentration that produces $50 \%$ of the maximal possible response for a given ligand. A highly potent ligand will evoke a certain response at low concentrations, while a ligand of lower potency will evoke the same response at much higher concentration.

The fitting of the FGF4 dose response curves presented a particular challenge, as we could only fit the increasing portions of the dose response curves to a rectangular hyperbolic. As described in Materials and Methods, we truncated the data for the fit, including the data in the ascending portions of the curves and taking into account that the average errors in the western blots are 5$10 \%$. In particular, we truncated all dose response curves at the highest ligand concentration that is within $10 \%$ of the maximum response value. Under the assumption that the decrease in the FGF4 dose response curve is due to oligomerization, the best-fit FGF4 dose response curves represent the response of the FGFR1 dimers to FGF4.

The best fits for all dose response curves are shown in Figure $2 \mathrm{C}$ with the solid lines. The best fit values of Etop and EC50 for all studied responses are shown in Table 1. FGF4 exhibits the highest potency, followed by either FGF8 or FGF9, dependent of the particular response. FGF8 exhibits the highest efficacy, followed by either FGF4 or FGF9, dependent of the particular response. In most cases, FGF8 is a full agonist, while FGF9 and FGF4 are partial agonists. However, FGF4 and FGF8 appear to be both full agonists when FRS2 phosphorylation is probed (see Table 1 and Figure 2C).

The results in Table 1 show that the rank-ordering of the different ligands is different when different responses are probed, which is indicative of ligand bias. "Biased agonism" or "ligand bias" is the ability of different ligands to differentially activate different signaling pathways downstream of the same receptor (45). Ligand bias reflects not just quantitative differences in downstream signaling, but a fundamental difference between the signaling responses to different ligands $(32,46)$. To determine if bias exists or not, we calculate bias coefficients using equation (2). Each bias coefficient, shown in Table 2, compares two responses and two ligands and reveals whether a response is preferentially engaged by one of the ligands (if $\beta \neq 0$ ) or whether 
both response are activated similarly by both ligands (if $\beta=0$ ). We refer to the entire set of coefficients as a "bias map."

We assessed statistical significance of the differences in bias coefficients for each pair of responses using ANOVA as described in Materials and Methods. The results of the statistical analysis are shown in Table 2, where gray shading indicates statistical significance between either FGF4 or FGF8, on one hand, and the reference ligand FGF8, on the other. We see no statistical significance between FGF4 and FGF9 (Supplemental Table S2).

Based on the ANOVA analysis, we conclude that FGF8 is biased towards phosphorylation of FRS2, against phosphorylation of Y653/654 and Y766, and against PLC $\gamma$ activation, as compared to FGF9. Furthermore, FGF8 is biased towards phosphorylation of FRS2 and against phosphorylation of Y653/654 and Y766, as compared to FGF4.

\section{Phosphorylation time courses cannot explain the existence of ligand bias}

Previously, it has been suggested that ligand bias may arise due to differences in the time courses of phosphorylation $(47,48)$. We therefore sought to compare phosphorylation of the activation loop tyrosines Y653/654 and FRS2 over time. We chose these two particular responses because, FGF8 is biased towards FRS2 and against Y653/654 FGFR1 phosphorylation, when compared to FGF4 and FGF9 (Table 2). Thus, we complemented the Y653/654 phosphorylation time course in Figure 3A, acquired at high concentration (130 nM FGF), with kinetics measurements of Y653/654 phosphorylation at low (2.6 nM) FGF concentration, as well as FRS2 phosphorylation at low (2.6 nM) and high (130 nM ligand) FGF concentrations. Three independent time courses were acquired for each ligand-receptor pair, and the results were averaged. Data, scaled such that the maximal phosphorylation is set to 1, are shown in Figure 3B-D.

We observe differences in the time courses of Y653/654 and FRS2 phosphorylation. In the case of FRS2, we always observe fast accumulation of phosphorylated FRS2, which then decreases over time. Such a behavior has been observed for other RTKs and can be explained by the fact that auto-phosphorylation within the RTK dimers/oligomers occurs faster than phosphatasemediated de-phosphorylation, after which a steady state is established (49, 50). An early maximum in phosphorylation (at 5 minutes) is seen for FRS2 at both low and high FGF concentration, and is very pronounced for high FGF concentration. An early phosphorylation 
maximum is also observed for Y653/654 FGFR at high FGF concentration, but not at low FGF concentration.

When comparing the time-courses of FGFR1 phosphorylation, in every panel of Figure 3, we do not see discernable differences in the time course of FGF8-induced phosphorylation, as compared to FGF4 or FGF9. Thus, the time course of phosphorylation alone cannot identify FGF8 as a biased ligand, when compared to FGF4 or FGF9.

\section{Differences in cellular responses to FGF8 and FGF9}

Biased signaling manifests itself in different cellular responses, which can be cell-specific (32). We therefore investigated if cells expressing FGFR1 respond differently when stimulated with FGF8 and FGF9, as these two ligands are biased and their effects at high concentrations can be directly compared as the phosphorylation responses come to a plateau (Figure 2). We compared FGFR1 clearance from the plasma membrane due to ligand-induced cell uptake, cell apoptosis, and cell viability, for the $293 \mathrm{~T}$ cells used in the western blotting experiments. These cellular responses have been previously reported to occur downstream of FGFR1 activation $(7,51,52)$.

To study FGFR1 endocytosis, we measured the decrease in FGFR1-YFP concentration in the plasma membrane in response to a 2-minute treatment of $130 \mathrm{nM}$ of FGF8 or FGF9. After fixation, the plasma membranes in contact with the substrate were imaged on a confocal microscope, and the fluorescence intensities for hundreds of cells stimulated with either FGF8, FGF9, or no ligand were recorded. The average fluorescence intensities for the three groups, from three independent experiments, are shown in Figure 4A. We observed a decrease in fluorescence, corresponding to a decrease in plasma membrane concentration of FGFR1-YFP in response to FGF8, as compared to FGF9 and no ligand. This effect was statistically significant by ANOVA $(\mathrm{p}<0.05)$. On the other hand, there were no statistically significant differences in FGFR1-YFP membrane concentrations between the FGF9-treated and control groups. These results indicate that FGF8 is more efficient at inducing FGFR1 removal from the plasma membrane immediately after ligand addition, as compared to FGF9. Of note, this result is consistent with the data in Figure 1C, which show that the efficacy of FGF8-induced downregulation, measured in the western blot experiments 20 minutes after ligand addition, was higher than the FGF9 efficacy. 
To monitor the apoptosis of cells exposed to different FGF8 and FGF9 concentrations, we used a commercial kit that measures the combined activity of caspases 3 and 7 (Magic Red caspase kit) through an increase in caspase substrate fluorescence. Experiments were conducted at 0, 20, 40, and $100 \mathrm{nM}$ FGF, under starvation conditions, and the results for each set of experiments were scaled such that caspase activity was 1.0 in the absence of FGF. Experiments were repeated at least five times. Data in Figure $4 \mathrm{~B}$ show the scaled caspase activity as a function of FGF concentration. We see a significant increase in caspase activity in the case of FGF8, but not FGF9. Data were fitted to linear functions, and the best-fit slopes were compared using a t-test. The difference between the slopes was statistically significant $(\mathrm{p}<0.005)$, indicating that FGF8 promotes apoptosis more efficiently than FGF9.

We next sought to measure and compare the relative amounts of live FGFR1-expressing cells after stimulation with ligand and after six days of starvation, using a MTT assay. The read-out as a function of ligand concentration is shown in Figure 4C, revealing a slight decrease with ligand concentration for both FGF8 and FGF9. Data were fitted to linear functions, and the slopes were compared using a t-test. Results show that there is a significant difference in cell viability when cells are stimulated with FGF8 or FGF9 $(\mathrm{p}<0.015)$, and the effects are significant when compared to the case of no ligand.

We thus observe that FGF8 is more efficient than FGF9 at promoting FGFR1 clearance from the plasma membrane, apoptosis, and the viability of cells under starvation conditions. This is a manifestation of ligand bias.

\section{Structural determinants behind FGFR1 biased signaling}

We sought possible structural determinants behind the observed FGF bias. For GPCRs, it is now well established that different GPCR ligands stabilize different receptor conformations, where each of the conformations has a preference for a subset of downstream signaling molecules (either $\mathrm{G}$ proteins or arrestins) $(22,53,54)$. We therefore asked if structural differences in the FGF4, FGF8, and FGF9-bound FGFR1 dimers may explain bias for FGF8, as compared to FGF4 and FGF9.

It is known that FRS2 binds to FGFR1 JM domain in a ligand-independent manner $(40,55)$. It is also believed that the conformation of the JM domain of RTKs is influenced by the conformation of the TM domain dimer in response to ligand binding $(54,56)$. Further, the FGFR1 TM domain 
has already been shown to sense the identity of the bound ligand, either FGF1 or FGF2, and adopt a different TM domain dimer conformation (22). We therefore sought to investigate the possibility that the TM domains of FGFR1 dimerize differently when FGF8, as compared to FGF4 and FGF9, is bound to the FGFR1 EC domain.

A Förster resonance energy transfer (FRET)-based methodology has been instrumental in revealing differences in RTK TM domain dimer conformations in response to ligand binding (22, $53,57)$. In these experiments, we monitored differences in the intracellular distances and relative orientation of fluorescent protein reporters when different ligands bind to the EC domain. We used truncated FGFR1 constructs, which contain the entire EC and TM domains of FGFR1 followed by a flexible linker and either mCherry or YFP. The fluorescent proteins mCherry and YFP are a FRET pair and thus report on the separation between the C-termini of the TM domains in a FGFR1 dimer (22). Measurements were performed in plasma membrane derived vesicles, as previously described (58). High concentration of ligand (250 nM) was used, to ensure that all receptors are ligand bound. We followed a quantitative protocol (termed QI-FRET) which yields (i) the FRET efficiency, (ii) the donor concentration, and (iii) the acceptor concentration in each vesicle (59). A few hundred vesicles, imaged in multiple independent experiments, were analyzed and the data were combined.

Figure 5A, left, shows the FRET efficiencies as a function of total FGFR1 concentration in the presence of the three ligands, where each data point corresponds to one individual vesicle derived from one cell. The total FGFR1 concentration, on the $\mathrm{x}$ axis in Figure 5A, left. is the sum of ECTM FGFR1-YFP and ECTM FGFR-mCherry concentrations. In Figure 5A, right, we further show the expressions of ECTM FGFR1-YFP and ECTM FGFR1-mCherry in the individual vesicles. Because transient expression levels vary from cell to cell, the vesicles produced in a single transfection experiment had a wide range of receptor concentrations.

We see that FRET in Figure 5A does not depend on the concentration, over a broad receptor expression range, indicative of constitutive FGFR1 association in the presence of the three FGF ligands. To interpret the FRET data, we need to know the oligomer size of the ECTM FGFR1 construct. FIF experiments (not shown) demonstrated that the ECTM construct always forms dimers, even in the presence of high FGF4 concentration. Thus, the data in Figure 5 correspond to ligand-bound ECTM FGFR1 dimers; in this case FRET depends only on (i) the fraction of 
acceptor-labeled FGFR1, which is directly calculated from the data in Figure 5A and (ii) the socalled Intrinsic FRET, a structural parameter which depends on the positioning and dynamics of the two fluorophores (60). An Intrinsic FRET value was calculated for each vesicle from the data in Figure 5A using equation 5. The values were binned to generate a histogram and are shown in Figure 5B. These histograms were fitted with Gaussians, yielding Intrinsic FRET values of 0.54 $\pm 0.01,0.42 \pm 0.01$ and $0.52 \pm 0.01$, for the cases of FGF4, FGF8, and FGF9, respectively. Thus, intrinsic FRET is lower for FGF8-bound ECTM FGFR1 dimers, as compared to FGF4 and FGF9-bound ECTM FGFR1 dimers. Since the fluorescent proteins were attached directly to the TM domains via flexible linkers, the measured differences in Intrinsic FRET reflect differences in the separation of the C-termini of the TM domains in the presence of FGF8, on one hand, and FGF4 and FGF9, in the other. Thus, the TM domain dimer structure is different in the FGF8 bound FGFR1 dimers as compared to FGF4 and FGF8 bound dimers.

The effective distances between the fluorescent proteins in the ECTM FGFR1 dimers are calculated using equation 6, assuming random orientation of the fluorophores (justified because they were attached via flexible 15 amino acid long linkers). In the presence of FGF4 and FGF9, the effective distance between the fluorescent proteins is the same, $55 \pm 1 \AA$ and $56 \pm 1 \AA$ (p > 0.05). In the presence of FGF8, the effective distance between the fluorescent proteins is significantly higher, $60 \pm 1 \AA$ ( $<<0.01)$ (Figure 5C).

\section{Discussion}

The most significant discovery in this work is the existence of bias in FGFR1 signaling in response to ligands that FGFR1 encounters during limb development. This concept of "ligand bias" is a relatively novel concept in RTK research (61). It describes the ability of ligands to differentially activate signaling pathways $(34,44,45)$. Ligand bias has been studied primarily in the context of G-protein coupled receptors (GPCRs) and has transformed the fundamental understanding of GPCR signaling (45). Importantly, these investigations have produced optimized protocols to identify and quantify bias that are directly applicable to RTKs as well (61). Here we use such quantitative protocols to demonstrate that FGF8 but not FGF4 or FGF9 preferentially induces FRS2 phosphorylation, when compared to the phosphorylation of FGFR1 tyrosines. 
Here we further observe that the bias in phosphorylation leads to different cellular responses. In particular, we find that FGF8 is more efficient than FGF9 at promoting FGFR1 removal from the plasma membrane and cellular apoptosis in HEK 293T cells. It is important to keep in mind that these responses are integrative, i.e. they depend on multiple signaling feed-back loops. They are thus dependent on the expression of downstream signaling molecules in the cell lines used, and more generally, on cellular context. In other cell types, biased signaling may manifest itself differently. FGFR1-induced functional responses have been previously studied in L6 myoblasts (62), PC12 cells $(63,64)$, hematopoetic $\mathrm{Ba} / \mathrm{F} 3$ cells $(38)$, and others. It will be interesting to compare FGFR1 signaling in response to FGF4, FGF8 and FGF9 in these well-studied cell lines too.

Having established bias in FGFR1 signaling, we sought the mechanism that allows for the recognition of FGF8 binding to FGFR1 EC domain, as compared to FGF4 and FGF9. This is a challenging question to answer, as the very mechanism of RTK signal propagation from the EC domains to the kinase domains is currently unknown. To a large extent, this is due to a complete lack of high-resolution structures of full-length RTKs. Furthermore, electron microscopy studies have unequivocally shown that there is no simple one-to-one correspondence between distinct EC and IC domain conformations $(65,66)$. This has led us to propose that every ligand-bound RTK dimer explores an ensemble of microstates, each characterized by different kinase domain dimer structures (67). The typical role of a microstate is to ensure the phosphorylation of one specific tyrosine. Additional microstates may serve as intermediates in the transitions between two phosphorylation-competent configurations, or may serve to recruit effector proteins by exposing phosphorylated tyrosines. The prevalence of a certain microstate (or its residence time, or its weight within the microstate ensemble) has been proposed to correlate with its stability, i.e., with the favorable contacts between the two chains in the full-length dimer (67). The fact that many different kinase domain conformations are experimentally observed (as multiple tyrosines are phosphorylated) suggests that multiple microstates have similar stabilities, and thus similar residency times. This view suggests that experimental observations of a specific kinase dimer conformation, as well as the entire ensemble of dimer conformations, will present enormous challenges.

A hypothesis about the mechanism of ligand bias postulates that structural differences in the EC region of an RTK bound to different ligands are propagated along the length of the RTK, 
resulting in differences in the interactions between the kinase domains $(22,53,68)$. This determines which cytoplasmic tyrosines are most efficiently phosphorylated, leading to the differential activation of downstream effectors (68). Given the challenges in resolving kinase dimer conformations discussed above, here we focused on the TM domains, which are simple helices that dimerize in the plasma membrane and impact signaling (69). We measured FRET occurring in truncated FGFR1 dimers with fluorescent proteins attached to the C-termini of the TM helices via flexible linkers. We showed that FRET is different when FGF8 is bound to the EC domain, as compared to the cases of bound FGF4 and FGF9. These results suggest that the C-termini of the TM helices are spaced further apart in the FGF8-bound FGFR1 dimers as compared to FGF4 and FGF9-bound dimers. Thus, FGFR1 TM domains must sense the identity of the ligand that is bound to the EC domain. FRS2 binds to the JM domain, which immediately follows the TM domain $(40,55,64)$. It is easy to envision that the conformation of the TM domain affects FRS2 behavior, as the FRS2 binding site is just 32 amino acids from the TM domain C-terminus. The larger TM domain separation in the FGF8-bound FGFR1 dimers may ensure the preferential phosphorylation of FRS2 over other sites. Alternatively, the smaller TM domain separation upon binding of FGF4 and FGF9 may inhibit FRS2 phosphorylation. Further, the differential phosphorylation of FRS2 may be due to a different mode of FRS2 binding to FGFR1, differences in the accessibility of FRS2 by the active site of the FGFR1 kinase, or different accessibility of FRS2 by phosphatases.

It is important to note that there are researchers who disagree that structural information can be propagated along the length of the RTK, because the linkers between the RTK domains are flexible $(47,70,71)$. If so, how do the kinases sense which ligand is bound to the EC domain? Some have proposed that differential downstream signaling occurs as a result of different kinetics of receptor phosphorylation/de-phosphorylation in response to different ligands (47). There is a report that tyrosine phosphorylation of EGFR is more sustained in response to epigen and epiregulin than to EGF(47). Specifically, it was found that EGF-induced EGFR phosphorylation of Y845, Y1086, and Y1173 returned to baseline much faster than when the cells were stimulated with epigen and epiregulin (47). The authors argued that differences in kinetics do not depend on the specific ligand concentration used, and they attributed the characteristic kinetics of the response to the identity of the bound ligand. In other studies with other receptors, however, the concentration of the ligand strongly influenced the kinetics of the 
response, questioning the applicability of this model to all RTKs $(72,73)$. Here we show that FGFR1 kinetics of phosphorylation also depend strongly on FGF concentration. Furthermore, we do not observe a discernable correlation between kinetics of FGFR1 phosphorylation and ligand bias, which provides further support for the importance of the FGFR1 dimer structure as a determinant of FGFR1 ligand bias.

We further see marked differences in the potencies of the three FGFs. Indeed, $50 \%$ of maximum phosphorylation is reached for $\sim 0.5 \mathrm{nM}$ FGF4, $\sim 2 \mathrm{nM}$ FGF9, and $\sim 10 \mathrm{nM}$ FGF8. Thus, the same level of phosphorylation of a specific tyrosine can be achieved for much lower concentrations of FGF4, as compared to FGF8 or FGF9. The relative EC50 values are expected to correlate with the binding affinities of the ligands for FGFR1. The reported dissociation constants, measured for FGF4/FGFR1 and FGF9/FGFR1 using surface plasmon resonance in the context of the isolated and truncated FGFR1 EC domains are $170 \mathrm{nM}$ and $1200 \mathrm{nM}$, respectively (74). Thus, FGF4 binds FGFR1 about an order of magnitude tighter than FGF9, consistent with the difference in potencies that we measure here.

We also observe differences in the efficacies of the three ligands. The highest phosphorylation is achieved in response to high concentrations of FGF8. FGF8 is a full agonist while FGF4 and FGF9 are partial agonists for most of the responses (with the exception of FRS2 phosphorylation). Along with differences in potencies and efficacies, we observe a significant difference in the very shape of the dose response curves. In particular, the FGF4 dose response does not follow the anticipated rectangular hyperbolic shape, unlike the cases of FGF8 and FGF9. We see that the FGF4-induced phosphorylation of FGFR1 and the effector molecules increases up to $\sim 3 \mathrm{nM}$ FGF4, and then gradually decreases as FGF4 concentration is further increased. The mechanism behind the decrease is unknown, but our data suggest that it cannot be explained with differential kinetics of de-phosphorylation/downregulation. Furthermore, there is no correlation between the unusual shape of the FGF4 dose response and ligand bias, as FGF4 is not biased when compared to FGF9. Instead, we see an intriguing correlation between the decrease in FGFR1 phosphorylation and the appearance of FGFR1 oligomers that are larger than dimers. Thus, we can explain the shape of the FGF4 dose response curves if we assume that phosphorylation is less efficient in FGFR1 oligomers as compared to FGFR1 dimers. It is important to note that another RTK, EGFR, has also been proposed to form oligomers in response to EGF, in addition to dimers $(75,76)$. EGFR dimers and oligomers have been 
proposed to have different activities $(75,76)$. It has been suggested that in the EGFR oligomer, each EGFR kinase may be able to phosphorylate and be phosphorylated by multiple neighboring kinases, leading to higher overall activation. Such a view, however, is not consistent with the FGFR1 data presented here. Perhaps, the functional role of FGFR1 oligomers is very different from that of the EGFR oligomers, as oligomerization seems to inhibit FGFR1 signaling.

In conclusion, we initiated this study in search of a possible difference in the response of FGFR1 to three FGF ligands. We found not one, but many differences. We observed quantitative differences in most aspects of the FGFR1 signaling response: ligand-induced oligomerization, potency, efficacy, and conformations of FGFR1 TM domain dimer. We also discovered the existence of ligand bias in FGFR1 signaling. The quantitative tools that we used were instrumental in revealing these differences, based on the analysis of FGFR 1 dose response curves for the three ligands. Of note, such dose response curves are typically collected for GPCRs (32, 77-79), but rarely for RTKs. Future use of such quantitative tools for other RTKs may similarly reveal unexpected differences in response to different ligands and may uncover exciting new biology.

All 58 RTKs have been implicated in many growth disorders and cancers $(1,69,80,81)$, and have been recognized as important drug targets. Inhibitors that specifically target the RTKs have been under development for decades now. Recent years have seen significant improvements in RTK-targeted molecular therapies, but these therapies have not yet fundamentally altered the survival and the quality of life of patients $(82,83)$. This may be because RTK signaling is much more complex than currently appreciated, as demonstrated here for FGFR1. Furthermore, it is possible that ligand bias plays an important role in RTK-linked pathologies. The practice of evaluating bias for novel RTK-targeting therapeutics will empower the discovery of a new generation of biased RTK inhibitors that selectively target pathogenic signaling pathways, and thus exhibit high specificity and low toxicity. 


\section{Materials and Methods}

\section{Cell Culture}

Human Embryonic kidney cells (HEK 293T), stably transfected with FGFR1-YFP, were grown in Dulbecco's modified eagle medium (Thermofisher, PN 31600034,) with 10\% fetal bovine serum and supplemented with 3.5 grams of glucose and 1.5 grams of sodium bicarbonate.

Chinese Hamster Ovarian (CHO) cells were grown in Dulbecco's modified eagle medium with $10 \%$ fetal bovine serum and supplemented with 0.8 grams of glucose and 1.5 grams of sodium bicarbonate and $1 \% \mathrm{NEAA}$ at $37^{\circ} \mathrm{C}$ and $5 \% \mathrm{CO} 2$.

\section{Western blots}

HEK 293T cells stably expressing FGFR1 were seeded in equal volumes into 12 well plates and allowed to grow to $\sim 70-90 \%$ confluency, while changing the media after 48 hours. The full media was aspirated and replaced with Dulbecco's modified eagle medium without FBS. Varying amounts of ligands (R\&D systems; FGF4, \#235-F4-025; FGF8, \#423-F8-025, and FGF9, \# 273-F9-025) were added to each well to create a ligand concentration gradient. Cells were incubated with the ligands at $37^{\circ} \mathrm{C}$ and $5 \% \mathrm{CO} 2$ for 20 minutes and then immediately placed on ice.

Media was aspirated and cells were immediately lysed with 2x Laemmli Buffer (BioRad 1610737) + 5\% BME. Lysates were moved to clean Eppendorf tubes and vortexed for 10 second intervals 6 times over the course of 5-10 minutes, staying on ice in the interim. Lysates were centrifuged, boiled at $98^{\circ} \mathrm{C}$ for $10 \mathrm{~min}$, and then immediately placed on ice until cool. Lysates were then centrifuged again and stored at $-20^{\circ} \mathrm{C}$ for later use. Lysates were thawed on ice and vortexed immediately prior to loading onto gel. Gels were run on ice at $140 \mathrm{~V}$ for 3 hours using Bio-Rad Mini-Protean TGX precast gels in 1X Tris/Gly/SDS running buffer (BioRad \#1610732). Gels were then equilibrated with transfer buffer (BioRad \#1610734) supplemented with 20\% menthanol for 10 min. Western transfer was performed using the Iblot 2 Gel Transfer Device (Thermofisher \#IB21001) and nitrocellulose packs (Thermofisher IB23001). Nitrocellulose membranes were removed and replaced with PVDF membranes (BioRad \#1620177) that had been activated in $100 \%$ methanol, all other steps were as prescribed by Thermofisher. Transfers were done at $25 \mathrm{~V}$ for 7 minutes. Following transfer, membranes were immediately trimmed and 
placed in either 5\% non-fat milk or 5\% BSA in 1X TBS supplemented with $1 \%$ Tween (Thermofisher \#1706475) (TBST) depending on the primary antibody used. Blocking was accomplished on a rocker for 1-2 hours at room temperature. Membranes were rinsed 2X with TBST and then primary antibody was added at a 1:1000 dilution. Primary antibodies (Cell Signaling: anti-Y653/654 \#3471S, anti-FGFR1 \#9740S, anti-pY766 FGFR1, \#2544, anti-pFRS2 \#3861S, anti-pPLC $\gamma$ \#2822, anti-PLC $\gamma$ 2821) were incubated with the membrane overnight at $4^{\circ} \mathrm{C}$ on a rocker. The primary antibody was removed and the membrane was washed with TBST 3 times for 5-15 min. The secondary antibody (Promega: anti-rabbit \#W4011) was added at a dilution of 1:10000 and allowed to incubate on a rocker at room temperature for 1-2 hours. The secondary antibody was removed and the membrane was washed with TBST 3x for 5-15 min. The membrane was incubated with chemiluminescent solution (Thermofisher Scientific West Femto Supersignal, \#1706435) and imaged on a Bio-Rad Gel-Doc XRS+.

Blot images were stored digitally. The intensity of each band was quantified using imageJ.

\section{Dose response curves}

The band intensities from at least 3 and up to 5 independent samples were quantified. The intensities were averaged and plotted as a function of ligand concentration for each response. The averaged dose response curves for the three ligands were scaled to each other. This was accomplished by re-running the samples that yielded the highest phosphorylation band intensities for a specific response on the same blot (Figure 2B). Intensities for each ligand on the common blot were averaged and the averages for the different ligands were scaled by setting the maximum value to 1 . The scaled dose response curves were fitted to a rectangular hyperbolic (Hill equation with $\mathrm{n}=1$ ) given by:

$$
\text { response }=\frac{[x] * E_{t o p}}{[x]+E C_{50}}
$$

Here $[\mathrm{x}]$ represents the concentration of ligand, while $\mathrm{E}_{\mathrm{top}}$ corresponds to the plateau at high ligand concentrations and $\mathrm{EC}_{50}$ corresponds to the ligand concentration value at which $50 \%$ of $\mathrm{E}_{\text {top }}$ is achieved.

To fit the data to eqn 1, we truncated each dose response curve at the highest ligand concentration that is within $10 \%$ of the maximum y value. This took into account that the 
average y value error is between 5-10\%. The data was fit in Mathematica with the Nonlinear Model fit function using the Levenberg-Marquardt minimization method, while allowing for a maximum of 100,000 iterations. The errors of the fit were weighted as the inverse of the square of the error.

\section{Calculation of bias coefficients}

To calculate bias coefficients, FGF8 was chosen as the reference ligand. Bias coefficients, $\beta$, for FGF4 and FGF9 were calculated using eqn 2.

$$
\beta_{4,9}=\log \left(\left(\frac{E_{t o p, 1}}{E C_{50,1}} * \frac{E C_{50,2}}{E_{t o p, 2}}\right)_{4,9} *\left(\frac{E C_{50,1}}{E_{t o p, 1}} * \frac{E_{t o p, 2}}{E C_{50.2}}\right)_{8}\right)
$$

Standard errors of $\beta$ are calculated using the standard errors of $\mathrm{EC}_{50}$ and $\mathrm{E}_{\mathrm{top}}$, as determined from the fit in eqn 1 , using the functional approach for multi-variable functions (84).

To compare bias coefficients for the three ligands and determine statistical significance in a three-way comparison, we follow the protocol in (61), re-writing eqn 2 as:

$$
\beta_{4,9}=\beta_{4,9}^{\prime}-\beta_{8}^{\prime}
$$

Where $\beta^{\prime}$ is calculated in equation 4 .

$$
\beta_{4,8,9}^{\prime}=\log \left(\frac{E_{t o p, 1}}{E C_{50,1}} * \frac{E C_{50,2}}{E_{t o p, 2}}\right)_{4,8,9}
$$

Standard errors of $\beta^{\prime}$ were calculated using the standard errors of $\mathrm{EC}_{50}$ and $\mathrm{E}_{\text {top }}$ using the functional approach for multi-variable functions (84). The $\beta^{\prime}$ values and their corresponding errors are reported in Table $\mathrm{S} 1$.

Statistical significance of the differences between $\beta$ ' values were calculated with a one-way ANOVA using the multi-variable analysis option in Prism. The data that were inputted were the mean, SEM, and $n$, the number of points contributing to the fit. $n=9$ for FGF4, $n=10$ for FGF8 and $n=7$ for FGF9. The calculated p-values are shown in Table S2.

\section{FRET measurements}

CHO cells were seeded into tissue culture-treated 6-well plates at a density of $2 * 10^{4}$ cells/well. 24 hours later, the cells were co-transfected with FGFR1-ECTM-eYFP and FGFR1-ECTMmCherry using Fugene HD (Promega \# E2311) according to the manufacturer's instructions. 
ECTM constructs included the entire extracellular domain and transmembrane domain up to residue 402 , followed by a (GGS) 5 flexible linker and the fluorophore (22). The amount of DNA as well as the donor to acceptor ratio of the DNA added was varied to achieve a wide range of donor and acceptor expressions (58).

Cells were vesiculated $\sim 24$ hours after transfection as described previously (85). Briefly, the cells were rinsed $2 \mathrm{x}$ with $30 \%$ PBS and then incubated for 13 hours at $37^{\circ} \mathrm{C}$ and $5 \% \mathrm{CO}_{2}$ in a chloride salt buffer. Vesicles were then transferred to 4-well glass-bottomed chamber slides for imaging on a Nikon confocal microscope with a 60x objective to capture images of the equatorial cross-sections of the vesicles in 3 channels: donor (eYFP), acceptor (mCherry), and FRET (53). FRET was measured following the QI-FRET protocol $(58,60)$, which yields the donor concentration, the acceptor concentration, and the FRET efficiency in each vesicle. The microscope was calibrated using solutions of fluorescent protein of known concentration, so that the fluorescence intensity could be directly correlated to fluorophore concentration.

In the case of constitutive dimers at high ligand concentration, when FRET does not depend on receptor concentration, the measured FRET and the Intrinsic FRET are connected as follows(59):

$$
\tilde{\mathrm{E}}=\frac{E}{x_{A}}
$$

eqn 5

Here $x_{\mathrm{A}}$ is the acceptor fraction, which is measured in each vesicle, along with the FRET efficiency, E. Equation 5 allows us to directly determine the value of the intrinsic FRET, Ẽ, in each vesicle. The dependence of the intrinsic FRET, $\tilde{\mathrm{E}}$, on the distance between the fluorescent proteins in the dimer, $d$, is given by equation (6).

$$
\tilde{\mathrm{E}}=\frac{1}{1+\left(\frac{d}{R_{O}}\right)^{6}}
$$

Here $R_{\mathrm{o}}$ is the Förster radius of the FRET pair. For eYFP and mCherry, $\mathrm{R}_{0}$ is $53.1 \AA(60,86)$. This equation assumes free rotation of the fluorescent proteins. This assumption is justified because the fluorescent proteins are attached via flexible linkers.

\section{Fluorescence Intensity Fluctuations (FIF) spectrometry}

HEK 293 T cells, stably transfected with FGFR1-YFP, were seeded on collagen-coated, glass bottom petri dishes (MatTek, P35GCOL-1.5-14-C) and allowed to grow to $70 \%$ confluency at 
$37^{\circ} \mathrm{C}$ and $5 \% \mathrm{CO}$. Cells were grown in Dulbecco's modified eagle medium with $10 \%$ fetal bovine serum, supplemented with 3.5 grams of glucose and 1.5 grams of sodium bicarbonate. Cells were rinsed with 70\% swelling media (1:9 serum-free media, diH2O, $25 \mathrm{mM}$ HEPES), and then swelled in $70 \%$ swelling media plus ligand for $\sim 5 \mathrm{~min}$ before imaging. This treatment minimizes the ruffles in the plasma membrane and prevents endocytosis $(87,88)$.

The plasma membranes facing the support were imaged on a TCS SP8 confocal microscope using a photon counting detector. Images were analyzed using the FIF software (23). Briefly, the membrane was divided into $15 \times 15$ pixel regions of interest and the molecular brightness of each region was calculated as:

$$
\varepsilon=\frac{\sigma^{2}}{\langle I\rangle}-I
$$

The brightness values from thousands of regions of interest were binned and histogrammed. The histograms were then normalized to 1 .

Since the molecular brightness distribution is log-normal, the values of $\log$ (brightness) were histogrammed and fit to a Gaussian function:

$$
\text { normalized counts }=a * e^{\frac{-(\theta-m)^{2}}{2 * s^{2}}}
$$

eqn 8

Here $\theta$ represents the $\log$ of the brightness, $\mathrm{m}$ is the mean of the Gaussian, $\mathrm{s}$ is the standard deviation of the Gaussian, and a is a constant. The best fit Gaussian parameters are shown in Table S3.

In order to determine whether the distributions were the same or different, a Z-statistics analysis was used, where the $\mathrm{Z}$ value is given by:

$$
Z=\frac{m_{1}-m_{2}}{\sqrt{q_{1}^{2}+q_{2}^{2}}}
$$

Here $\mathrm{m}_{1}$ and $\mathrm{m}_{2}$ represent the two means of the Gaussians being compared, and $\mathrm{q}_{1}$ and $\mathrm{q}_{2}$ are the standard deviations for each Gaussian divided by the square root of the number of cells analyzed. A minimum of 100 cells were analyzed for each data set. 
A $\mathrm{Z}$ value of less than 2 means that the data sets are within 2 standard deviations of the mean and are therefore the same, while a $\mathrm{Z}$ value greater than 2 means that the two data sets are different (31). Calculated $\mathrm{Z}$ values can be found in Table S3.

\section{Ligand-induced FGFR1 removal from the plasma membrane}

FGFR1 downregulation in the plasma membrane was assayed by measuring the FGFR1 membrane concentration before and 2 minutes after ligand addition. FGFR1-YFP in the plasma membrane in contact with the substrate was imaged in a TCS SP8 confocal microscope, and FGFR1-YFP fluorescence per unit membrane area was quantified. HEK 293T cells, stably transfected with FGFR1-YFP, were seeded on collagen-coated, glass bottom petri dishes (MatTek, P35GCOL-1.5-14-C) and allowed to grow to $\sim 70 \%$ confluency at $37^{\circ} \mathrm{C}$ and $5 \% \mathrm{CO} 2$. Cells were rinsed with serum-free media and exposed to $130 \mathrm{nM}$ of either FGF8 or FGF9 or no ligand and incubated at $37^{\circ} \mathrm{C}$ and $5 \% \mathrm{CO} 2$ for 2 minutes. The cells were fixed in a solution of $4 \%$ formaldehyde in PBS for $20 \mathrm{~min}$ at room temperature. Samples were stored at $4^{\circ} \mathrm{C}$ prior to imaging. A minimum of 100 cells per condition were imaged, in three independent experiments. The receptor concentration in the membrane of each cell was quantified using the FIF software (23), and results for all analyzed cells per condition were averaged.

\section{Apoptosis Assays}

Apoptosis was probed using the Bio-Rad Magic Red Caspase 3-7 kit (Bio-Rad, ICT 935). HEK 293T cells stably expressing FGFR1-YFP were seeded in 96 well plates and allowed to grow to $\sim 70 \%$ confluency. Media was aspirated and replaced with serum-free media with the Magic Red staining solution and varying concentrations of ligand. After ligand treatment the cells were placed in the incubator at $37^{\circ} \mathrm{C}$ and $5 \% \mathrm{CO} 2$ for 6 days. The fluorescence of the cleaved substrate (Cresyl Violet) was measured on a Synergy H4 plate reader. The excitation wavelength was $592 \mathrm{~nm}$. Emission was measured at $628 \mathrm{~nm}$.

\section{Viability Assays}

Cell viability was monitored using a MTT Cell Proliferation Assay Kit (Cell BioLabs, \#CBS252). HEK 293 T cells, stably transfected with FGFR1-YFP, were seeded in 96 well plates and allowed to grow to $\sim 70 \%$ confluency. Media was aspirated and replaced with serum-free media and with varying concentrations of ligand. After ligand addition, cells were kept at $37^{\circ} \mathrm{C}$ and $5 \%$ 
$\mathrm{CO} 2$ for 6 days. Viability was measured according to the manufacturer's protocol. Briefly, CytoSelect MTT Cell Proliferation Assay Reagent was added directly to the cell media. After three hours of incubation at $37^{\circ} \mathrm{C}$ and $5 \% \mathrm{CO} 2$, the cells were lysed with detergent and kept at room temperature for 2 hours. The absorbance was measured on a Synergy H4 plate reader at $555 \mathrm{~nm}$.

\section{Acknowledgement}

Supported by NIH GM068619 and NSF MCB 2106031. PK is supported by the Agency for Healthcare Research of the Czech Republic (NV18-08-00567), the Czech Science Foundation (GA19-20123S), and the Grand Agency of Masaryk University (MUNI/G/1771/2020).

\section{References}

1. Lemmon, M. A., and J. Schlessinger. 2010. Cell Signaling by Receptor Tyrosine Kinases. Cell 141:1117-1134.

2. Schlessinger, J. 2000. Cell signaling by receptor tyrosine kinases. Cell 103:211-225.

3. Paul, M. D., and K. Hristova. 2019. The RTK Interactome: Overview and Perspective on RTK Heterointeractions. Chem Rev 119:5881-5921.

4. Goetz, R., and M. Mohammadi. 2013. Exploring mechanisms of FGF signalling through the lens of structural biology. Nat.Rev.Mol.Cell Biol. 14:166-180.

5. Eswarakumar, V. P., I. Lax, and J. Schlessinger. 2005. Cellular signaling by fibroblast growth factor receptors. Cytokine Growth Factor Rev. 16:139-149.

6. Schlessinger, J. 2004. Common and distinct elements in cellular signaling via EGF and FGF receptors. Science 306:1506-1507.

7. Tomlinson, D. C., F. R. Lamont, S. D. Shnyder, and M. A. Knowles. 2009. Fibroblast growth factor receptor 1 promotes proliferation and survival via activation of the mitogen-activated protein kinase pathway in bladder cancer. Cancer Res 69:4613-4620.

8. Ornitz, D. M., and N. Itoh. 2001. Fibroblast growth factors. Genome Biology 2.

9. Ornitz, D. M., J. S. Xu, J. S. Colvin, D. G. McEwen, C. A. MacArthur, F. Coulier, G. X. Gao, and M. Goldfarb. 1996. Receptor specificity of the fibroblast growth factor family. Journal of Biological Chemistry 271:15292-15297.

10. Zhang, X. Q., O. A. Ibrahimi, S. K. Olsen, H. Umemori, M. Mohammadi, and D. M. Ornitz. 2006. Receptor specificity of the fibroblast growth factor family - The complete mammalian FGF family. Journal of Biological Chemistry 281:15694-15700.

11. Buchtova, M., R. Chaloupkova, M. Zakrzewska, I. Vesela, P. Cela, J. Barathova, I. Gudernova, R. Zajickova, L. Trantirek, J. Martin, M. Kostas, J. Otlewski, J. Damborsky, A. Kozubik, A. Wiedlocha, and P. Krejci. 2015. Instability restricts signaling of multiple fibroblast growth factors. Cellular and Molecular Life Sciences 72:2445-2459.

12. Passos-Bueno, M. R., A. L. Sertie, A. Richieri-Costa, L. G. Alonso, M. Zatz, N. Alonso, D. Brunoni, and S. F. M. Ribeiro. 1998. Description of a new mutation and 
characterization of FGFR1, FGFR2, and FGFR3 mutations among Brazilian patients with syndromic craniosynostoses. American Journal of Medical Genetics 78:237-241.

13. Wilkie, A. O. M. 2005. Bad bones, absent smell, selfish testes: The pleiotropic consequences of human FGF receptor mutations. Cytokine \& Growth Factor Reviews 16:187-203.

14. White, K. E., J. M. Cabral, S. I. Davis, T. Fishburn, W. E. Evans, S. Ichikawa, J. Fields, X. J. Yu, N. J. Shaw, N. J. McLellan, C. McKeown, D. FitzPatrick, K. Yu, D. M. Ornitz, and M. J. Econs. 2005. Mutations that cause osteoglophonic dysplasia define novel roles for FGFR1 in bone elongation. American Journal of Human Genetics 76:361-367.

15. Turner, N., A. Pearson, R. Sharpe, M. Lambros, F. Geyer, M. A. Lopez-Garcia, R. Natrajan, C. Marchio, E. Iorns, A. Mackay, C. Gillett, A. Grigoriadis, A. Tutt, J. S. Reis, and A. Ashworth. 2010. FGFR1 Amplification Drives Endocrine Therapy Resistance and Is a Therapeutic Target in Breast Cancer. Cancer Research 70:2085-2094.

16. Seo, A. N., Y. Jin, H. J. Lee, P. L. Sun, H. Kim, S. Jheon, K. Kim, C. T. Lee, and J. H. Chung. 2014. FGFR1 amplification is associated with poor prognosis and smoking in non-small-cell lung cancer. Virchows Archiv 465:547-558.

17. Mariani, F. V., and G. R. Martin. 2003. Deciphering skeletal patterning: clues from the limb. Nature 423:319-325.

18. Tabin, C., and L. Wolpert. 2007. Rethinking the proximodistal axis of the vertebrate limb in the molecular era. Genes Dev 21:1433-1442.

19. Mariani, F. V., C. P. Ahn, and G. R. Martin. 2008. Genetic evidence that FGFs have an instructive role in limb proximal-distal patterning. Nature 453:401-U456.

20. Needham, S. R., S. K. Roberts, A. Arkhipov, V. P. Mysore, C. J. Tynan, L. C. ZanettiDomingues, E. T. Kim, V. Losasso, D. Korovesis, M. Hirsch, D. J. Rolfe, D. T. Clarke, M. D. Winn, A. Lajevardipour, A. H. Clayton, L. J. Pike, M. Perani, P. J. Parker, Y. Shan, D. E. Shaw, and M. L. Martin-Fernandez. 2016. EGFR oligomerization organizes kinase-active dimers into competent signalling platforms. Nat Commun 7:13307.

21. Singh, D. R., P. Kanvinde, C. King, E. B. Pasquale, and K. Hristova. 2018. The EphA2 receptor is activated through induction of distinct, ligand-dependent oligomeric structures. Communications Biology 1:15.

22. Sarabipour, S., and K. Hristova. 2016. Mechanism of FGF receptor dimerization and activation. Nat.Commun. 7:10262.

23. Stoneman, M. R., G. Biener, R. J. Ward, J. D. Pediani, D. Badu, A. Eis, I. Popa, G. Milligan, and V. Raicu. 2019. A general method to quantify ligand-driven oligomerization from fluorescence-based images. Nat Methods 16:493-496.

24. Digman, M. A., and E. Gratton. 2012. Scanning image correlation spectroscopy. BioEssays 34:377-385.

25. Paul, M. D., R. Rainwater, Y. Zuo, L. Gu, and K. Hristova. 2021. Probing Membrane Protein Association Using Concentration-Dependent Number and Brightness. Angew Chem Int Ed Engl 60:6503-6508.

26. Paul, M. D., H. N. Grubb, and K. Hristova. 2020. Quantifying the strength of heterointeractions among receptor tyrosine kinases from different subfamilies: Implications for cell signaling. The Journal of biological chemistry 295:9917-9933.

27. Ahmed, F., E. Zapata-Mercado, S. Rahman, and K. Hristova. 2021. The Biased Ligands NGF and NT-3 Differentially Stabilize Trk-A Dimers. Biophys J 120:55-63. 
28. Moscatelli, D. 1987. High and Low Affinity Binding-Sites for Basic Fibroblast GrowthFactor on Cultured-Cells - Absence of A Role for Low Affinity Binding in the Stimulation of Plasminogen-Activator Production by Bovine Capillary Endothelial-Cells. Journal of Cellular Physiology 131:123-130.

29. Roghani, M., A. Mansukhani, P. Dell'Era, P. Bellosta, C. Basilico, D. B. Rifkin, and D. Moscatelli. 1994. Heparin increases the affinity of basic fibroblast growth factor for its receptor but is not required for binding. The Journal of biological chemistry 269:39763984.

30. Gleizes, P. E., J. Noaillac-Depeyre, F. Amalric, and N. Gas. 1995. Basic fibroblast growth factor (FGF-2) internalization through the heparan sulfate proteoglycansmediated pathway: an ultrastructural approach. Eur J Cell Biol 66:47-59.

31. Anderson, D., D. J. Sweeney, T. A. Williams, J. D. Camm, and J. J. Cochran. 2001. Modern Business Statistics with Microsoft Excel.

32. Kenakin, T. 2019. Biased Receptor Signaling in Drug Discovery. Pharmacol Rev 71:267315.

33. Kenakin, T. 2017. Signaling bias in drug discovery. Expert Opin Drug Discov 12:321333.

34. Smith, J. S., R. J. Lefkowitz, and S. Rajagopal. 2018. Biased signalling: from simple switches to allosteric microprocessors. Nat Rev Drug Discov 17:243-260.

35. Wisler, J. W., H. A. Rockman, and R. J. Lefkowitz. 2018. Biased G Protein-Coupled Receptor Signaling: Changing the Paradigm of Drug Discovery. Circulation 137:23152317.

36. Furdui, C. M., E. D. Lew, J. Schlessinger, and K. S. Anderson. 2006. Autophosphorylation of FGFR1 kinase is mediated by a sequential and precisely ordered reaction. Molecular Cell 21:711-717.

37. Lew, E. D., C. M. Furdui, K. S. Anderson, and J. Schlessinger. 2009. The precise sequence of FGF receptor autophosphorylation is kinetically driven and is disrupted by oncogenic mutations Science Signaling 2:ra6.

38. Sorokin, A., M. Mohammadi, J. Huang, and J. Schlessinger. 1994. Internalization of fibroblast growth factor receptor is inhibited by a point mutation at tyrosine 766 . The Journal of biological chemistry 269:17056-17061.

39. Burgar, H. R., H. D. Burns, J. L. Elsden, M. D. Lalioti, and J. K. Heath. 2002. Association of the signaling adaptor FRS2 with fibroblast growth factor receptor 1 (Fgfr1) is mediated by alternative splicing of the juxtamembrane domain. The Journal of biological chemistry 277:4018-4023.

40. Gotoh, N. 2008. Regulation of growth factor signaling by FRS2 family docking/scaffold adaptor proteins. Cancer Sci 99:1319-1325.

41. Huang, Z. F., W. M. Marsiglia, U. B. Roy, N. Rahimi, D. Ilghari, H. Y. Wang, H. B. Chen, W. M. Gai, S. Blais, T. A. Neubert, A. Mansukhani, N. J. Traaseth, X. K. Li, and M. Mohammadi. 2016. Two FGF Receptor Kinase Molecules Act in Concert to Recruit and Transphosphorylate Phospholipase C gamma. Molecular Cell 61:98-110.

42. Rajagopal, S., S. Ahn, D. H. Rominger, W. Gowen-MacDonald, C. M. Lam, S. M. Dewire, J. D. Violin, and R. J. Lefkowitz. 2011. Quantifying ligand bias at seventransmembrane receptors. Mol Pharmacol 80:367-377.

43. Kenakin, T., and A. Christopoulos. 2013. Measurements of ligand bias and functional affinity. Nat Rev Drug Discov 12:483. 
44. Kenakin, T. 2016. Measurement of Receptor Signaling Bias. Curr Protoc Pharmacol 74:2 15 11-12 1515.

45. Gundry, J., R. Glenn, P. Alagesan, and S. Rajagopal. 2017. A Practical Guide to Approaching Biased Agonism at G Protein Coupled Receptors. Front Neurosci 11:17.

46. Ehlert, F. J. 2018. Analysis of Biased Agonism. Prog Mol Biol Transl Sci 160:63-104.

47. Freed, D. M., N. J. Bessman, A. Kiyatkin, E. Salazar-Cavazos, P. O. Byrne, J. O. Moore, C. C. Valley, K. M. Ferguson, D. J. Leahy, D. S. Lidke, and M. A. Lemmon. 2017. EGFR Ligands Differentially Stabilize Receptor Dimers to Specify Signaling Kinetics. Cell 171:683-695 e618.

48. Kiyatkin, A., I. K. van Alderwerelt van Rosenburgh, D. E. Klein, and M. A. Lemmon. 2020. Kinetics of receptor tyrosine kinase activation define ERK signaling dynamics. Sci Signal 13.

49. Suwanmajo, T., and J. Krishnan. 2013. Biphasic responses in multi-site phosphorylation systems. J R Soc Interface 10:20130742.

50. Lax, I., A. Wong, B. Lamothe, A. Lee, A. Frost, J. Hawes, and J. Schlessinger. 2002. The docking protein FRS2alpha controls a MAP kinase-mediated negative feedback mechanism for signaling by FGF receptors. Mol Cell 10:709-719.

51. Xie, Y., N. Su, J. Yang, Q. Tan, S. Huang, M. Jin, Z. Ni, B. Zhang, D. Zhang, F. Luo, H. Chen, X. Sun, J. Q. Feng, H. Qi, and L. Chen. 2020. FGF/FGFR signaling in health and disease. Signal Transduct Target Ther 5:181.

52. Xian, W., K. L. Schwertfeger, and J. M. Rosen. 2007. Distinct roles of fibroblast growth factor receptor 1 and 2 in regulating cell survival and epithelial-mesenchymal transition. Mol Endocrinol 21:987-1000.

53. Sarabipour, S., K. Ballmer-Hofer, and K. Hristova. 2016. VEGFR-2 conformational switch in response to ligand binding. Elife 5.

54. Doerner, A., R. Scheck, and A. Schepartz. 2015. Growth Factor Identity Is Encoded by Discrete Coiled-Coil Rotamers in the EGFR Juxtamembrane Region. Chem.Biol. 22:776784.

55. Ong, S. H., G. R. Guy, Y. R. Hadari, S. Laks, N. Gotoh, J. Schlessinger, and I. Lax. 2000. FRS2 proteins recruit intracellular signaling pathways by binding to diverse targets on fibroblast growth factor and nerve growth factor receptors. Mol Cell Biol 20:979-989.

56. Scheck, R. A., M. A. Lowder, J. S. Appelbaum, and A. Schepartz. 2012. Bipartite tetracysteine display reveals allosteric control of ligand-specific EGFR activation. ACS Chem Biol 7:1367-1376.

57. Ahmed, F., and K. Hristova. 2018. Dimerization of the Trk receptors in the plasma membrane: effects of their cognate ligands. The Biochemical journal 457:3669-3685.

58. Sarabipour, S., N. Del Piccolo, and K. Hristova. 2015. Characterization of Membrane Protein Interactions in Plasma Membrane Derived Vesicles with Quantitative Imaging Forster Resonance Energy Transfer. Acc.Chem.Res. 48:2262-2269.

59. King, C., M. Stoneman, V. Raicu, and K. Hristova. 2016. Fully quantified spectral imaging reveals in vivo membrane protein interactions. Integr.Biol.(Camb.) 8:216-229.

60. Chen, L. R., L. Novicky, M. Merzlyakov, T. Hristov, and K. Hristova. 2010. Measuring the Energetics of Membrane Protein Dimerization in Mammalian Membranes. Journal of the American Chemical Society 132:3628-3635.

61. Karl, K., M. D. Paul, E. B. Pasquale, and K. Hristova. 2020. Ligand bias in receptor tyrosine kinase signaling. The Journal of biological chemistry 295:18494-18507. 
62. Mohammadi, M., C. A. Dionne, W. Li, N. Li, T. Spivak, A. M. Honegger, M. Jaye, and J. Schlessinger. 1992. Point mutation in FGF receptor eliminates phosphatidylinositol hydrolysis without affecting mitogenesis. Nature 358:681-684.

63. Spivakkroizman, T., M. A. Lemmon, I. Dikic, J. E. Ladbury, D. Pinchasi, J. Huang, M. Jaye, G. Crumley, J. Schlessinger, and I. Lax. 1994. Heparin-Induced Oligomerization of Fgf Molecules Is Responsible for Fgf Receptor Dimerization, Activation, and CellProliferation. Cell 79:1015-1024.

64. Hadari, Y. R., H. Kouhara, I. Lax, and J. Schlessinger. 1998. Binding of Shp2 tyrosine phosphatase to FRS2 is essential for fibroblast growth factor-induced PC12 cell differentiation. Molecular and Cellular Biology 18:3966-3973.

65. Mi, L. Z., C. Lu, Z. Li, N. Nishida, T. Walz, and T. A. Springer. 2011. Simultaneous visualization of the extracellular and cytoplasmic domains of the epidermal growth factor receptor. Nat Struct Mol Biol 18:984-989.

66. Opatowsky, Y., I. Lax, F. Tome, F. Bleichert, V. M. Unger, and J. Schlessinger. 2014. Structure, domain organization, and different conformational states of stem cell factorinduced intact KIT dimers. Proc Natl Acad Sci U S A 111:1772-1777.

67. Karl, K., and K. Hristova. 2021. Pondering the mechanism of receptor tyrosine kinase activation: The case for ligand-specific dimer microstate ensembles. Curr Opin Struct Biol 71:193-199.

68. Wilson, K. J., J. L. Gilmore, J. Foley, M. A. Lemmon, and D. J. Riese. 2009. Functional selectivity of EGF family peptide growth factors: Implications for cancer. Pharmacology \& Therapeutics 122:1-8.

69. Li, E., and K. Hristova. 2006. Role of receptor tyrosine kinase transmembrane domains in cell signaling and human pathologies. Biochemistry 45:6241-6251.

70. Lu, C., L. Z. Mi, M. J. Grey, J. Zhu, E. Graef, S. Yokoyama, and T. A. Springer. 2010. Structural evidence for loose linkage between ligand binding and kinase activation in the epidermal growth factor receptor. Mol Cell Biol 30:5432-5443.

71. Bocharov, E. V., K. S. Mineev, K. V. Pavlov, S. A. Akimov, A. S. Kuznetsov, R. G. Efremov, and A. S. Arseniev. 2017. Helix-helix interactions in membrane domains of bitopic proteins: Specificity and role of lipid environment. Biochim Biophys Acta 1859:561-576.

72. Wykosky, J., E. Palma, D. M. Gibo, S. Ringler, C. P. Turner, and W. Debinski. 2008. Soluble monomeric EphrinA1 is released from tumor cells and is a functional ligand for the EphA2 receptor. Oncogene 27:7260-7273.

73. Gomez-Soler, M., M. Petersen Gehring, B. C. Lechtenberg, E. Zapata-Mercado, K. Hristova, and E. B. Pasquale. 2019. Engineering nanomolar peptide ligands that differentially modulate EphA2 receptor signaling. The Journal of biological chemistry 294:8791-8805.

74. Mohammadi, M., S. K. Olsen, and O. A. Ibrahimi. 2005. Structural basis for fibroblast growth factor receptor activation. Cytokine \& Growth Factor Reviews 16:107-137.

75. Needham, S. R., S. K. Roberts, A. Arkhipov, V. P. Mysore, C. J. Tynan, L. C. ZanettiDomingues, E. T. Kim, V. Losasso, D. Korovesis, M. Hirsch, D. J. Rolfe, D. T. Clarke, M. D. Winn, A. Lajevardipour, A. H. Clayton, L. J. Pike, M. Perani, P. J. Parker, Y. Shan, D. E. Shaw, and M. L. Martin-Fernandez. 2016. EGFR oligomerization organizes kinase-active dimers into competent signalling platforms. Nat Commun 7. 
76. Huang, Y., S. Bharill, D. Karandur, S. M. Peterson, M. Marita, X. Shi, M. J. Kaliszewski, A. W. Smith, E. Y. Isacoff, and J. Kuriyan. 2016. Molecular basis for multimerization in the activation of the epidermal growth factor receptor. Elife 5:e14107.

77. Luttrell, L. M. 2014. Minireview: More than just a hammer: ligand "bias" and pharmaceutical discovery. Mol Endocrinol 28:281-294.

78. Correll, C. C., and B. A. McKittrick. 2014. Biased ligand modulation of seven transmembrane receptors (7TMRs): functional implications for drug discovery. J Med Chem 57:6887-6896.

79. Kenakin, T. 2011. Functional selectivity and biased receptor signaling. J Pharmacol Exp Ther 336:296-302.

80. Robertson, S. C., J. A. Tynan, and D. J. Donoghue. 2000. RTK mutations and human syndromes - when good receptors turn bad. Trends in Genetics 16:265-271.

81. Browne, B. C., N. O'Brien, M. J. Duffy, J. Crown, and N. O'Donovan. 2009. HER-2 Signaling and Inhibition in Breast Cancer. Current Cancer Drug Targets 9:419-438.

82. Le, X., M. Nilsson, J. Goldman, M. Reck, K. Nakagawa, T. Kato, L. P. Ares, B. FrimodtMoller, K. Wolff, C. Visseren-Grul, J. V. Heymach, and E. B. Garon. 2021. Dual EGFRVEGF Pathway Inhibition: A Promising Strategy for Patients With EGFR-Mutant NSCLC. J Thorac Oncol 16:205-215.

83. Kumar, R., B. George, M. R. Campbell, N. Verma, A. M. Paul, C. Melo-Alvim, L. Ribeiro, M. R. Pillai, L. M. da Costa, and M. M. Moasser. 2020. HER family in cancer progression: From discovery to 2020 and beyond. Adv Cancer Res 147:109-160.

84. Hughes, I. G., and T. Hase. 2010. Measurements and their Uncertainties: A practical guide to modern error analysis.

85. Del Piccolo, N., J. Placone, L. He, S. C. Agudelo, and K. Hristova. 2012. Production of plasma membrane vesicles with chloride salts and their utility as a cell membrane mimetic for biophysical characterization of membrane protein interactions. Analytical Chemistry 84 8650-8655.

86. Chen, L., J. Placone, L. Novicky, and K. Hristova. 2010. The extracellular domain of fibroblast growth factor receptor 3 inhibits ligand-independent dimerization. Science Signaling 3:ra86.

87. Rauch, C., and E. Farge. 2000. Endocytosis switch controlled by transmembrane osmotic pressure and phospholipid number asymmetry. Biophys J 78:3036-3047.

88. Sinha, B., D. Koster, R. Ruez, P. Gonnord, M. Bastiani, D. Abankwa, R. V. Stan, G. Butler-Browne, B. Vedie, L. Johannes, N. Morone, R. G. Parton, G. Raposo, P. Sens, C. Lamaze, and P. Nassoy. 2011. Cells respond to mechanical stress by rapid disassembly of caveolae. Cell. 144:402-413. 
Table 1: Best fit parameters for dose response curves in Figure 2. EC50 is the potency of the ligand, and Etop is the efficacy (see equation 1)

\begin{tabular}{|l|l|l|l|l|l|}
\hline \multicolumn{2}{|c|}{ pY653/654 } & \multicolumn{3}{c|}{ pPLC $\gamma$} & Etop \\
\hline & EC50 & Etop & & EC50 & $0.80 \pm 0.04$ \\
\hline FGF4 & $4.77 \mathrm{E}-10 \pm 0.42 \mathrm{E}-10$ & $0.95 \pm 0.03$ & FGF4 & $7.98 \mathrm{E}-11 \pm 2.15 \mathrm{E}-11$ & $0.99 \pm 0.03$ \\
\hline FGF8 & $1.04 \mathrm{E}-8 \pm 0.23 \mathrm{E}-8$ & $1.11 \pm 0.07$ & FGF8 & $1.05 \mathrm{E}-9 \pm 0.23 \mathrm{E}-9$ & \multicolumn{1}{c|}{ pFRS2 } \\
\hline FGF9 & $2.09 \mathrm{E}-9 \pm 0.31 \mathrm{E}-9$ & $0.70 \pm 0.02$ & FGF9 & $2.05 \mathrm{E}-10 \pm 0.40 \mathrm{E}-10$ & $0.62 \pm 0.02$ \\
\hline \multicolumn{7}{|c|}{ pY766 } & Etop & & EC50 & Etop \\
\hline & EC50 & & & $0.94 \pm 0.06$ \\
\hline FGF4 & $5.84 \mathrm{E}-10 \pm 1.26 \mathrm{E}-10$ & $0.76 \pm 0.05$ & FGF4 & $3.42 \mathrm{E}-10 \pm 0.70 \mathrm{E}-10$ & $0.98 \pm 0.03$ \\
\hline FGF8 & $1.42 \mathrm{E}-8 \pm 0.25 \mathrm{E}-8$ & $1.16 \pm 0.07$ & FGF8 & $1.62 \mathrm{E}-9 \pm 0.29 \mathrm{E}-9$ & $0.52 \pm 0.01$ \\
\hline FGF9 & $1.94 \mathrm{E}-9 \pm 0.71 \mathrm{E}-9$ & $0.56 \pm 0.05$ & FGF9 & $1.65 \mathrm{E}-9 \pm 0.15 \mathrm{E}-9$ & \\
\hline \multicolumn{7}{|c|}{ Downregulation } & & & & \\
\hline & EC50 & Etop & & & \\
\hline FGF4 & $7.34 \mathrm{E}-10 \pm 4.54 \mathrm{E}-10$ & $0.27 \pm 0.04$ & & & \\
\hline FGF8 & $1.39 \mathrm{E}-8 \pm 0.39 \mathrm{E}-8$ & $0.63 \pm 0.05$ & & & \\
\hline FGF9 & $6.80 \mathrm{E}-9 \pm 2.08 \mathrm{E}-9$ & $0.42 \pm 0.02$ & & & \\
\hline
\end{tabular}

Table 2: Calculated Bias Coefficients using equation 2. Gray shading indicates statistical significance between either FGF4 or FGF9 and the reference ligand FGF8. We see no statistical significance between FGF4 and FGF9 (Supplemental Table S2).

\begin{tabular}{|c|c|c|}
\hline \multicolumn{3}{|c|}{$\boldsymbol{\beta}$} \\
\hline & $4 v 8$ & $9 \mathrm{v8}$ \\
\hline pY653/654 vs pY766 & $0.07 \pm 0.16$ & $0.05 \pm 0.15$ \\
\hline pY653/654 vs pPLC $\gamma$ & $0.24 \pm 0.18$ & $0.01 \pm 0.14$ \\
\hline pY653/654 vs pFRS2 & $0.61 \pm 0.16$ & $-0.78 \pm 0.12$ \\
\hline pY766 vs pPLC $\gamma$ & $0.18 \pm 0.19$ & $-0.04 \pm 0.17$ \\
\hline pY766 vs pFRS2 & $0.54 \pm 0.17$ & $-0.83 \pm 0.16$ \\
\hline pPLC $\gamma$ vs pFRS2 & $0.37 \pm 0.20$ & $-0.79 \pm 0.13$ \\
\hline pY653/654 vs DownRegulation & $0.36 \pm 0.26$ & $-0.36 \pm 0.16$ \\
\hline pY766 vs DownRegulation & $0.29 \pm 0.27$ & $-0.41 \pm 0.19$ \\
\hline pPLC $\gamma$ vs DownRegulation & $0.12 \pm 0.28$ & $-0.37 \pm 0.16$ \\
\hline pFRS2 vs DownRegulation & $-0.25 \pm 0.27$ & $0.42 \pm 0.15$ \\
\hline
\end{tabular}



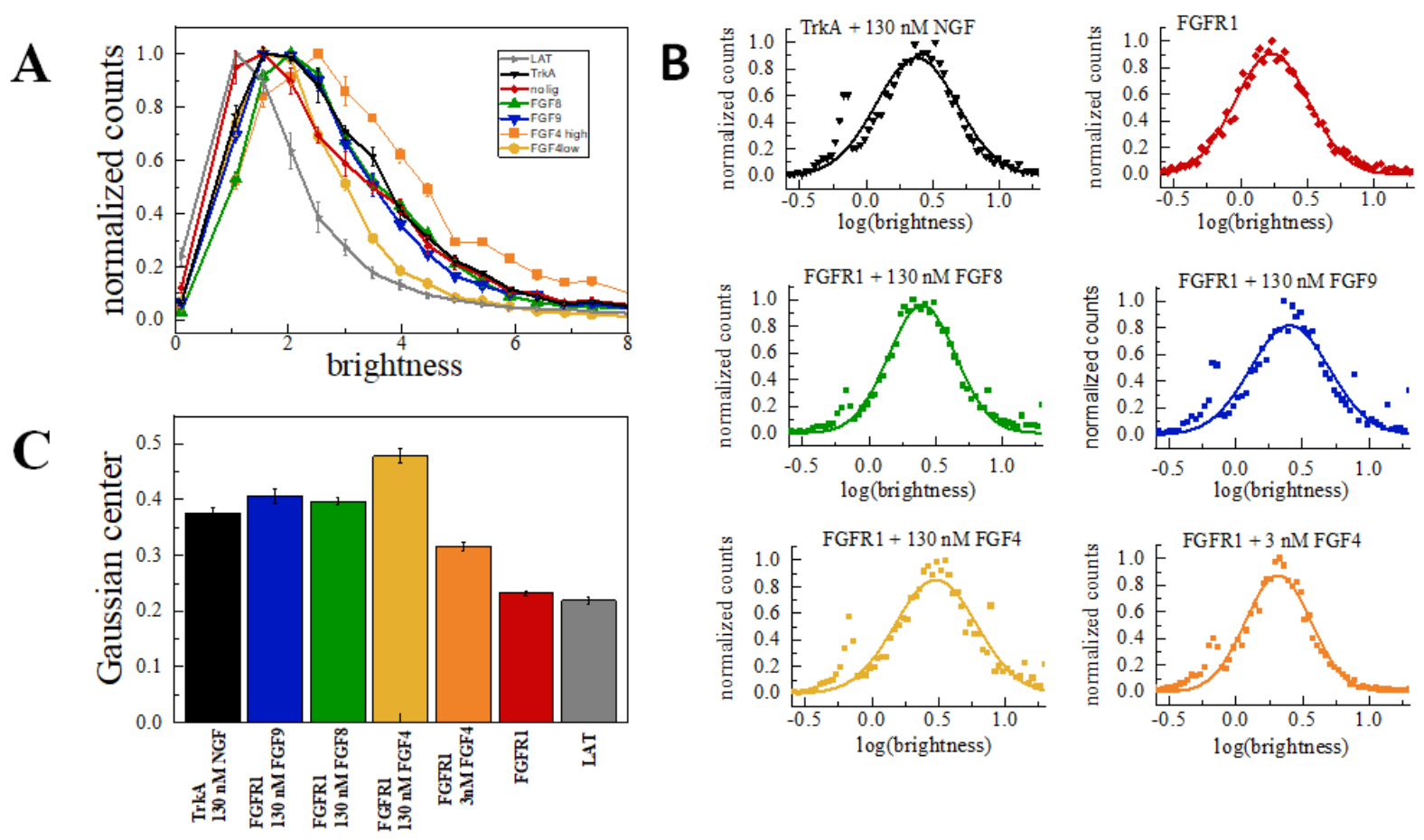

Figure 1: The oligomerization state of FGFR1, as measured by fluorescence intensity fluctuation (FIF) spectrometery. (A) Brightness distributions shown on the linear scale. Brightness scales with the oligomer size. LAT (gray) is a monomer control, TrkA+130 nM NGF (black) is a dimer control. All distributions are scaled to a maximum of 1. (B) Distributions of $\log$ (brightness). Points represent the experimental FIF data, and the solid lines are the best fit Gaussians. (C) Means of the best-fit Gaussians and the standard errors of the mean. 

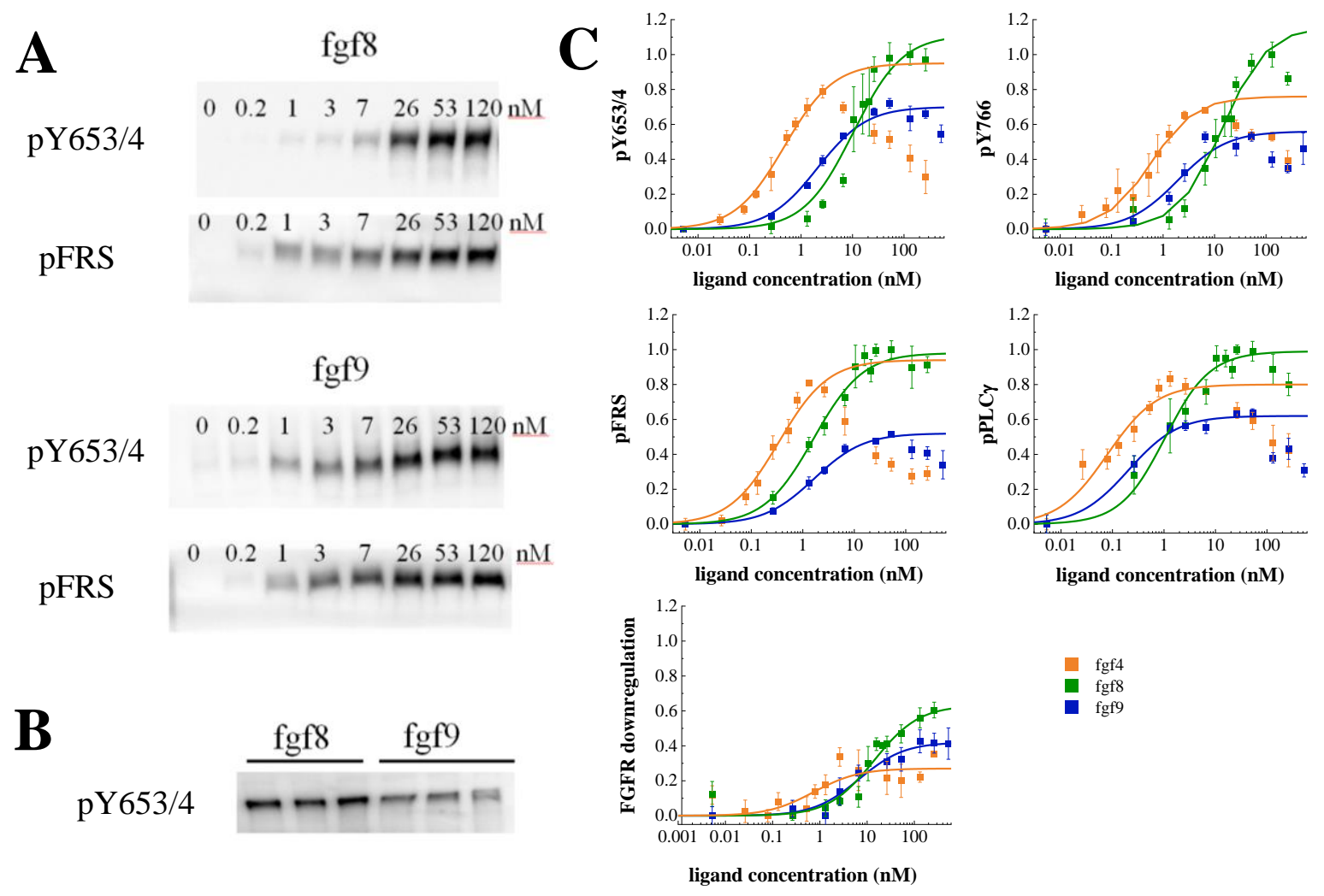

Figure 2: Activation of FGFR1 and downstream signaling substrates. (A) Sample western blots for Y653/4 FGFR1 phosphorylation and FRS2 phosphorylation in response to FGF8 and FGF9. (B) An example blot used for data scaling, where samples with maximum phosphorylation in response to FGF8 and FGF9 are rerun on the same gel (C) Dose response curves from the Western blot experiments. The points represent the averaged data, while the solid lines are the best fit rectangular hyperbolic curves. Fit parameters are shown in Table 1. 

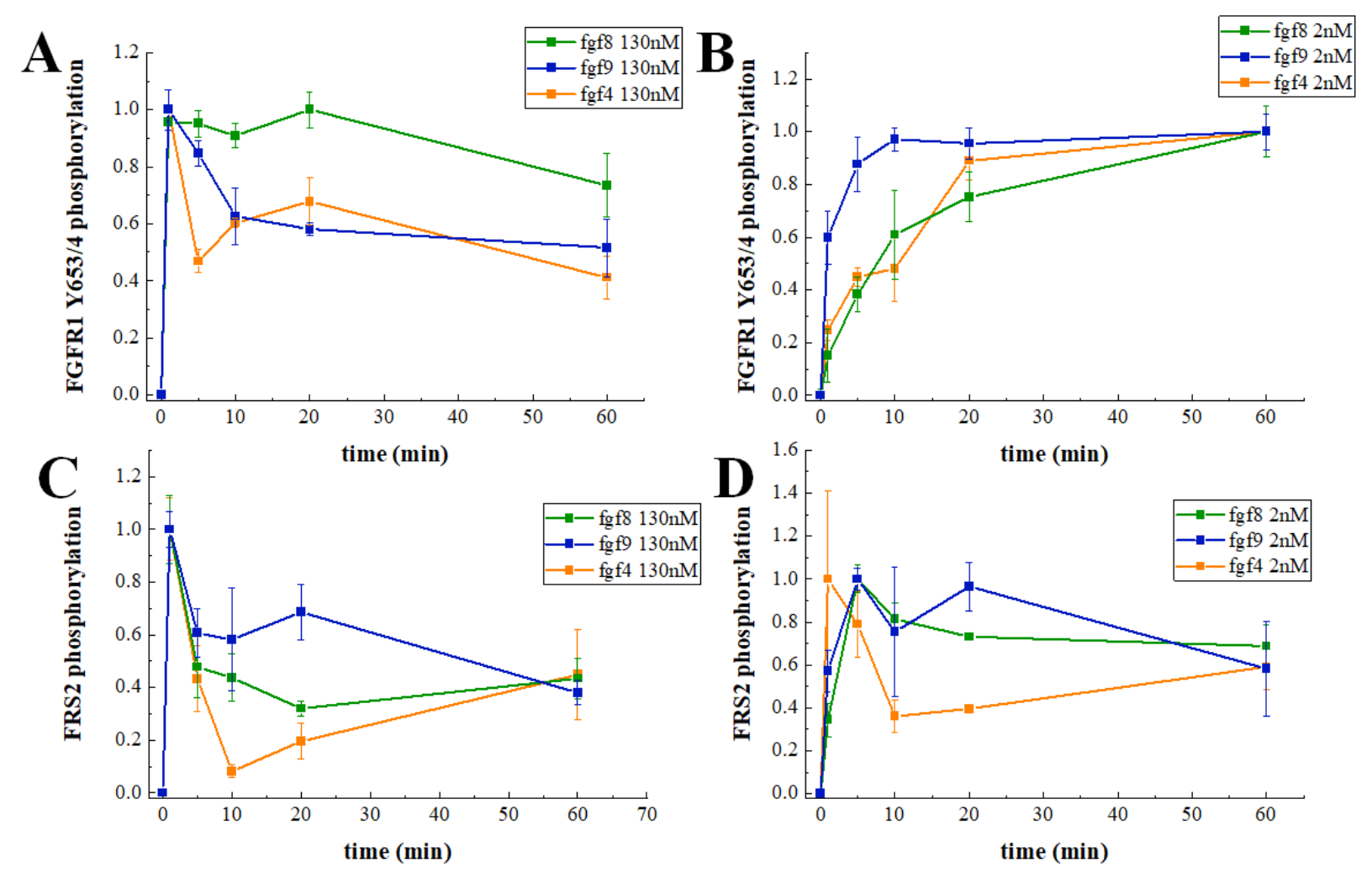

Figure 3: FGFR1 phosphorylation as a function of time after ligand addition. (A) Phosphorylation time course of Y653/654 at high ligand concentration (130 nM) (B) Phosphorylation time-course of Y653/654 at low ligand concentration (2.6 nM) (C) Phosphorylation time-course of FRS2 at high ligand concentration (130 nM) (B) Phosphorylation time-course of FRS2 at low ligand concentration (2.6 nM) 


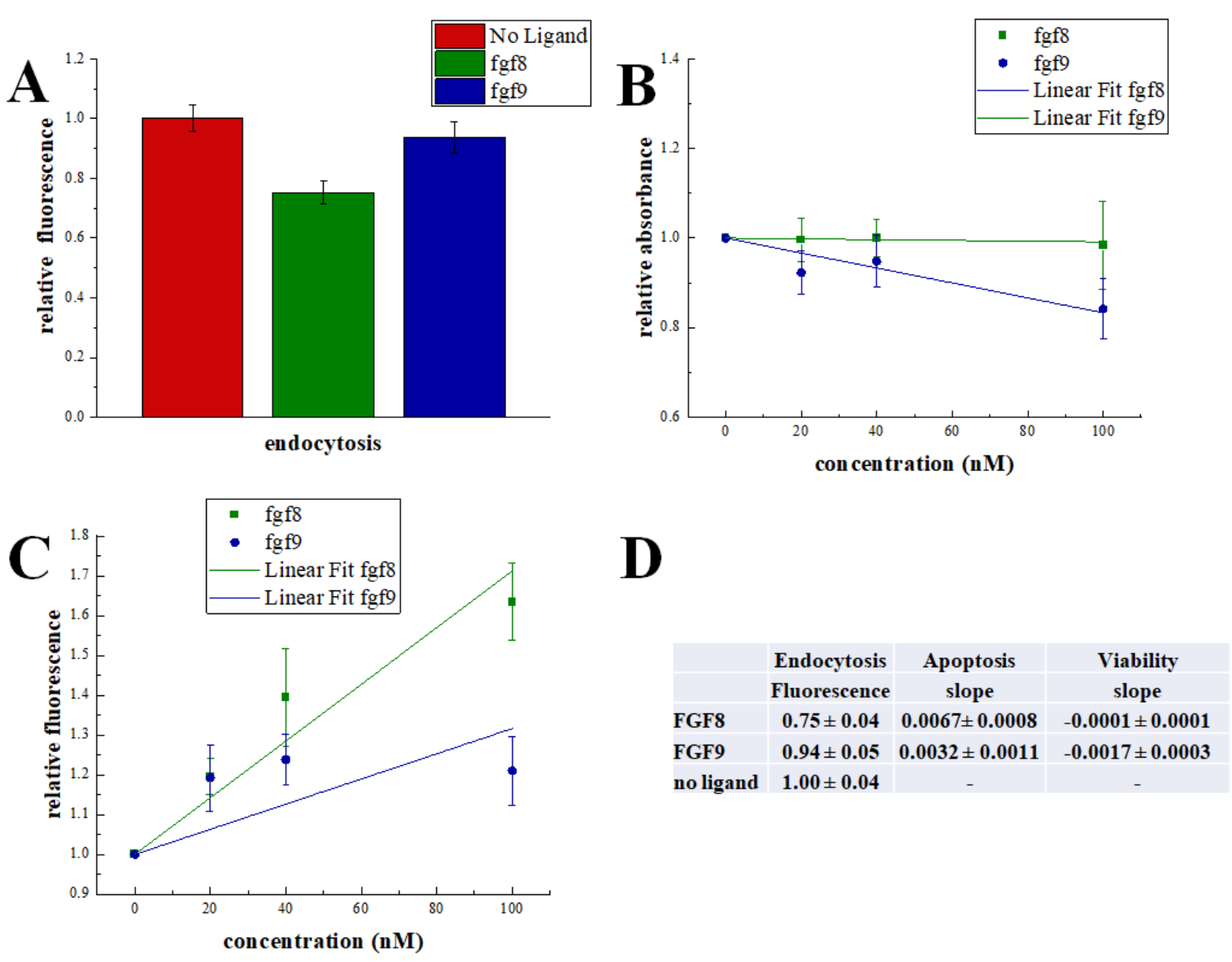

Figure 4: Responses of stably transfected HEK 293T cells expressing FGFR1 to the ligands FGF8 and FGF9. (A) FGFR1 concentration in the plasma membrane at $\mathrm{t}=2$ mins following ligand addition for FGF8, FGF9 and no ligand control. (B) Apoptosis of cells under starvation conditions, exposed to varying concentrations of FGF8 and FGF9. (C) Cell viability after ligand exposure and six days of starvation for varying ligand concentrations. (D) Table summarizing the results. 

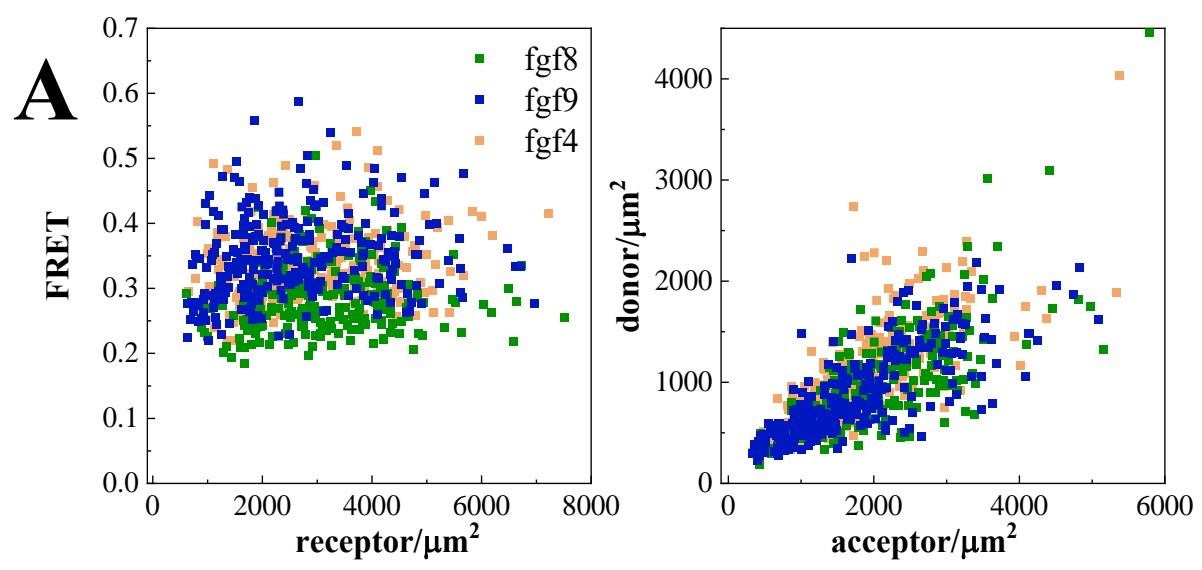

B
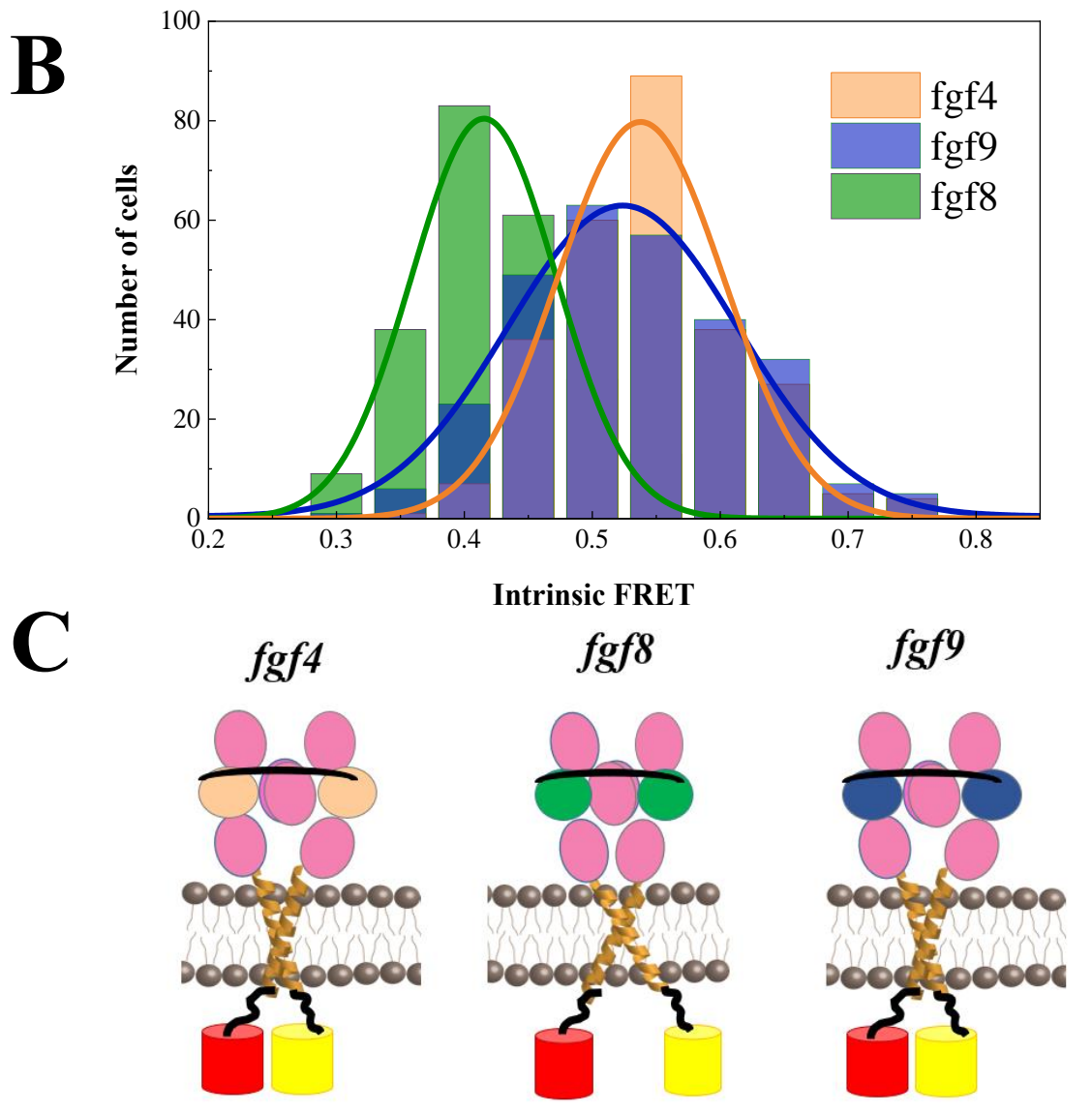
Figure 5: FRET Data for ECTM-FGFR1-YFP and ECTM-FGFR1-mCherry in the presence of saturating FGF4 (orange), FGF8 (blue) or FGF9 (green) concentrations. A) Measured FRET efficiencies versus total receptor (ECTM-FGFR1-YFP + ECTM-FGFR1-mCherry) concentrations and measured donor (ECTMFGFR1-YFP) concentrations versus acceptor (ECTM-FGFR1-YFP) concentrations in single vesicles. (B) Histograms of single-vesicle intrinsic FRET values. Intrinsic FRET is a measure of the separation between the fluorescent proteins in the dimer. Different intrinsic FRET values were measured for FGF8 and FGF4/FGF9. (C) Graphical representation of experimental results showing that FGF8 induces a TM conformation where the fluorophores are positioned further apart, as compared to the cases of FGF4 and FGF9. 